\title{
Resolution of type 2 diabetes following gastric bypass surgery: involvement of gut-derived glucagon and glucagonotropic signalling?
}

\author{
F. K. Knop
}

Received: 30 January 2009 / Accepted: 28 July 2009 /Published online: 1 September 2009

(C) Springer-Verlag 2009

\begin{abstract}
Certain types of bariatric surgical procedures have proved not only to be effective with regard to treating obesity, but they also seem to be associated with endocrine changes which independently of weight loss give rise to remission of type 2 diabetes. Currently, it is speculated that surgical re-routing of nutrients triggers changes in the release of gastrointestine-derived hormones, which in turn cause amelioration of the diabetic state. The 'hindgut hypothesis' states that surgical re-routing of nutrients to the distal part of the small intestine results in increased secretion and concomitant glucose-lowering effects of glucagon-like peptide-1, whereas the 'foregut hypothesis' emphasises that surgical bypass of the foregut prevents the release of a hitherto unidentified nutrient-induced diabetogenic signal in susceptible individuals. Recent studies have shown that in patients with type 2 diabetes, glucagon is differentially secreted in response to oral and i.v. glucose, respectively, with lack of suppression (and initial net secretion) during oral glucose administration and a perfectly normal suppression during isoglycaemic i.v. glucose administration. These findings could point towards a role for glucagon or gut-derived glucagonotropic signalling as putative diabetogenic signals of the foregut hypothesis. In the present paper the hypotheses describing the glucoselowering mechanisms of bariatric surgical procedures sharing the common feature of a bypass of the duodenum
\end{abstract}

\section{F. K. Knop ( $ه)$}

Diabetes Research Division, Department of Internal Medicine F, Gentofte Hospital, University of Copenhagen,

Niels Andersens Vej 65,

2900 Hellerup, Denmark

e-mail: filipknop@dadlnet.dk and the proximal jejunum are outlined and a possible role for glucagon in these is proposed.

Keywords Bariatric surgery Biliopancreatic diversion . Glucagon · Glucagon-like peptide-1 · Glucagon-like peptide-2 . Glucose-dependent insulinotropic polypeptide $\cdot$ Incretin hormones $\cdot$ Roux-en-Y gastric bypass $\cdot$ Type 2 diabetes

\author{
Abbreviations \\ DJB Duodenal jejunal bypass \\ GIP Glucose-dependent insulinotropic polypeptide \\ GJ Gastrojejunostomy \\ GK Goto-Kakizaki \\ GLP-1 Glucagon-like peptide-1 \\ GLP-2 Glucagon-like peptide-2 \\ LAGB Laparascopic adjustable gastric banding \\ PC1 Prohormone convertase 1 \\ PC2 Prohormone convertase 2 \\ RYGB Roux-en-Y gastric bypass
}

\section{Introduction}

Bariatric surgery has become the most effective therapeutic modality for the treatment of type 2 diabetes in overweight patients [1]. Besides the positive effect of weight loss on glucose metabolism, certain types of bariatric surgical procedures entail anatomical re-routing of nutrients through the gastrointestinal tract - a re-routing which in itself is thought to be the most significant contributor to postoperative resolution of the diabetic state. The present debate paper briefly outlines the present understanding of the 
'acute' glucose-lowering effects associated with bariatric surgical procedures that involve bypass of the proximal part of the small intestine, and suggests an additional explanation of the current hypotheses.

\section{Bariatric surgery: mechanisms of diabetes resolution}

The Roux-en-Y gastric bypass (RYGB) procedure (Fig. 1) leads to resolution of the diabetic state in $>80 \%$ of obese patients with type 2 diabetes [1]. Interestingly, the majority of patients undergoing bariatric surgical procedures which include a bypass of the duodenum and proximal jejunum, as opposed to, for example, laparascopic adjustable gastric banding (LAGB), where only a restrictive change is introduced, experience resolution of diabetes within days postoperatively long before the surgical procedure has caused any significant weight loss [1]. At present two hypotheses have been proposed to explain this rapid resolution of type 2 diabetes: the 'hindgut hypothesis' [2] and the 'foregut hypothesis' [3]. The former is based upon the ability of expedited delivery of ingested nutrients to the distal part of the small intestine to enhance the secretion of factors improving glucose metabolism; whereas the latter advocates the glucose-lowering effect to depend on exclusion of the duodenum and proximal jejunum from transit of nutrients.

\section{The hindgut hypothesis}

A number of facts lend support to the hindgut hypothesis. First of all a candidate molecule with immense blood sugarlowering power exists: glucagon-like peptide-1 (GLP-1). It is produced in mucosal endocrine L cells (found in the epithelium of the intestinal tract with highest density in the ileum) and secreted in response to nutrients in the intestinal lumen. GLP-1 has strong glucose-dependent insulinotropic properties and has been shown to enhance all steps of insulin biosynthesis as well as insulin gene transcription [4]. In addition, GLP-1 upregulates the genes for the cellular machinery involved in insulin secretion, such as the glucokinase and GLUT-2 (also known as SLC2A2) genes [5]. Importantly, GLP-1 also has trophic effects on beta cells: it stimulates beta cell proliferation, enhances the differentiation of new beta cells from pancreatic progenitor cells, and inhibits apoptosis of beta cells [6]. Furthermore, GLP-1 robustly inhibits glucagon secretion, and the combined effects on insulin and glucagon secretion result in inhibition of hepatic glucose production [7]. In addition, GLP-1 reduces appetite and gastrointestinal motility, lowering postprandial glucose excursions [8]. It is feasible that surgically induced expedited delivery of nutrients to the $L$ cell-rich distal part of the small intestine increases postprandial GLP-1 responses and brings about GLP-1's

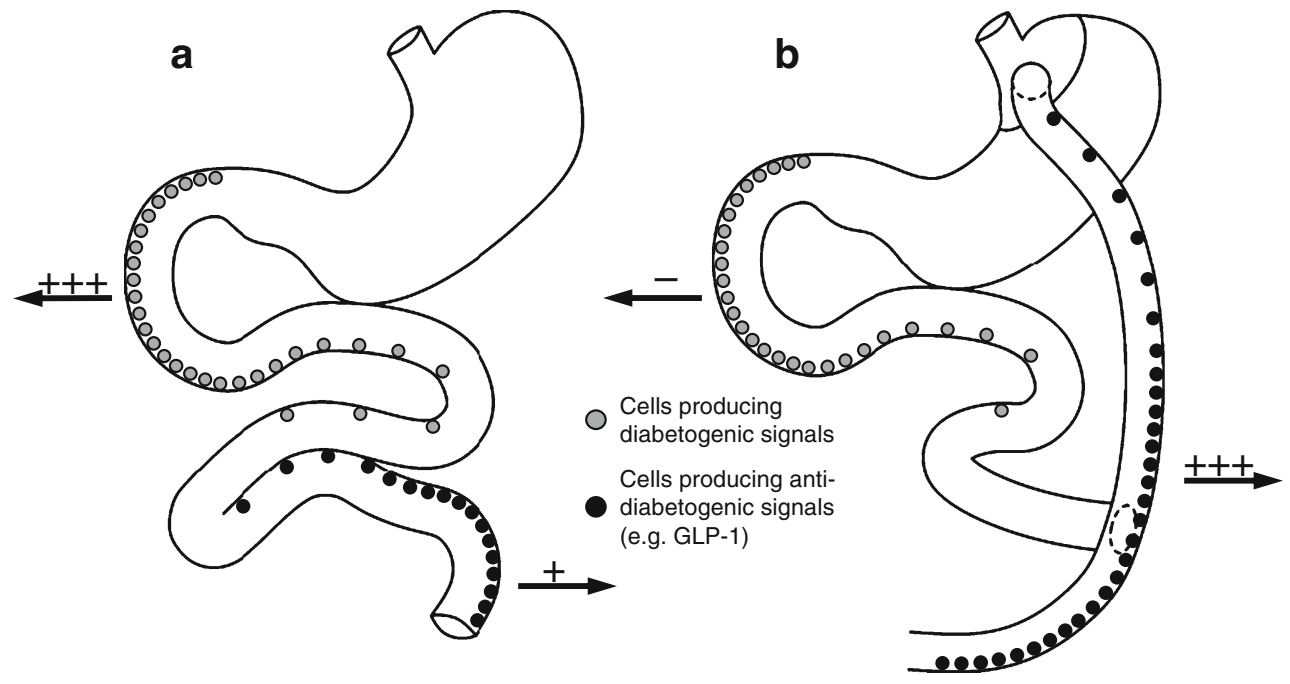

Fig. 1 a The normal anatomy of the stomach, duodenum and jejunum shown schematically. The 'foregut hypothesis' proposes that when the foregut of susceptible individuals is overstimulated with nutrients it releases a diabetogenic signal resulting in the development of type 2 diabetes. b Anatomical changes produced by RYGB are indicated. These include division of the stomach into a small upper pouch and a much larger, lower 'remnant' pouch, division of the small bowel about $45 \mathrm{~cm}$ below the lower stomach outlet, and re-arrangement into a $Y$ configuration, enabling outflow of food from the small upper stomach pouch, via a 'Roux limb'. The Y intersection is formed $80-150 \mathrm{~cm}$ from the anastomosis between the small upper gastric pouch and the small bowel. The foregut hypothesis emphasises that following
RYGB, diabetogenic signalling is avoided explaining the 'acute' RYGB-induced resolution of type 2 diabetes. The elaboration of the foregut hypothesis presented in the present paper suggests that part of the 'foregut' diabetogenic signals consist of glucagon itself and/or glucagonotropic signalling (e.g. by the hormone GIP) (indicated by ' +++ ' in a), which, in susceptible individuals, during normal anatomical conditions, would result in increased hepatic glucose output and subsequent hyperglycaemia. Following RYGB, these signals would be circumvented (indicated by ',' in b) and therefore, in combination with increased anti-diabetogenic signalling (i.e. GLP-1 secretion; indicated by ' +++ ' in b) from the hindgut, result in amelioration of the diabetic state 
glucose-lowering effects. Therefore, GLP-1 constitutes an obvious candidate molecule for the hindgut hypothesis. In line with this, GLP-1 responses to mixed meals or OGTTs have consistently been found to be exaggerated following RYGB, biliopancreatic diversion or jejunoileal bypass, which all expedite the transit of nutrients to the hindgut [9-12].

\section{The foregut hypothesis}

In 1998 Hickey et al. [13] proposed the hypothesis that type 2 diabetes is due to excessive production of a 'diabetogenic' signal generated in the proximal part of the small intestine. Pories et al. [14] elaborated this hypothesis and proposed that bariatric surgical procedures that include a bypass of the duodenum and proximal jejunum would prevent ingested nutrients from eliciting a 'diabetogenic signal' from the proximal part of the small intestine and hence ameliorate the diabetic condition. Rubino and colleagues $[3,15]$ elaborated this hypothesis and named it the 'foregut hypothesis'. In the non-obese rat model of type 2 diabetes, the Goto-Kakizaki (GK) rat, Rubino and Marescaux performed duodenal jejunal bypass (DJB) operations and observed significant improvements in glucose tolerance compared with sham-operated rats, despite equal body weights [16]. In another study in GK diabetic rats, Rubino et al. investigated the foregut hypothesis and the hindgut hypothesis by comparing the results of DJB (testing the foregut hypothesis) and a simple gastrojejunostomy (GJ) that supposedly allows rapid delivery of nutrients to the distal part of the small intestine and at the same time preserved nutrient passage to the duodenum and proximal part of the small intestine (testing the hindgut hypothesis) [15]. The investigators confirmed that exclusion of the proximal small intestine is a critical component in the amelioration of the diabetic state after DJB in GK rats, and showed first that GJ, as opposed to DJB, was not able improve glucose tolerance, second that surgical exclusion of duodenal passage of nutrients in GK diabetic rats that had initially undergone GJ improved glucose tolerance, and finally that restoration of duodenal passage in animals initially operated with DJB made these animals return to a 'diabetic state'. Rubino and colleagues propose that when the foregut of susceptible individuals is overstimulated with carbohydrates and fat it releases an unknown factor which contributes to the development of type 2 diabetes [3, 15]. Following surgical bypass of the foregut, release of this putative diabetogenic signal is avoided, explaining the prompt resolution of type 2 diabetes (Fig. 1).

Studies from our group indicate that a possible candidate molecule for the putative diabetogenic signal in the foregut hypothesis may be a well-known diabetogenic hormone: glucagon.

\section{Abnormal regulation of glucagon secretion in type 2 diabetes}

Patients with type 2 diabetes display abnormal regulation of glucagon secretion, with high fasting plasma glucagon levels and lack of glucagon suppression, or even net secretion, following ingestion of glucose loads or meals [17]. Fasting hyperglucagonaemia sustains glucose overproduction in the liver, contributing to type 2 diabetic fasting hyperglycaemia, and lack of postprandial glucagon suppression in diabetic patients contributes to the exaggerated glucose excursions following oral ingestion of glucose or meals [18]. Furthermore, studies in first-degree relatives of patients with type 2 diabetes suggest that inappropriately controlled glucagon secretion plays a role in the early aetiology of type 2 diabetes [19].

\section{A surprising finding}

The author of this paper has participated in the investigation of glucagon responses to OGGT and isoglycaemic i.v. glucose infusion (i.e. two situations with identical plasma glucose concentrations with and without glucose stimulation of the gastrointestinal tract) in different forms of diabetes (including type 2 diabetes) and in matched healthy control individuals $[20,21]$. In healthy individuals glucagon responses during the two glucose stimuli are suppressed equally. In patients with diabetes, an expected lack of glucagon suppression during oral glucose is observed (in fact the patients exhibit an initial net secretion of glucagon), whereas, surprisingly, a completely normal suppression of glucagon occurs during isoglycaemic i.v. glucose administration bypassing the gastrointestinal tract. An Italian group have reported similar observations [22] and a German group have reported a less-pronounced version of the phenomenon in healthy individuals [23]. Owing to the isoglycaemic conditions during the two stimuli, it is unlikely that differences in blood glucose are the cause of the heterogeneic glucagon response. In the mentioned studies, most insulin was secreted in response to the OGTT, which therefore, contrary to the results, should lead to a more pronounced suppression of glucagon succeeding oral glucose. One explanation of the heterogeneic glucagon responses takes as its starting point the different method of administration of glucose, and suggests that intestinederived mechanisms elicited by the oral ingestion of glucose play a role in the inappropriate glucagon suppression in patients with diabetes. Such mechanisms would be 
bypassed during i.v. administration of glucose, explaining the perfectly normal suppression of glucagon during isoglycaemic i.v. glucose infusion in diabetic patients.

Even though hyperglucagonaemia has been known to be an important pathophysiological characteristic of type 2 diabetes for more than 30 years, the mechanisms underlying this pathophysiological trait remain obscure. In this paper it is proposed that (1) intestine-derived mechanisms are crucial for postprandial hyperglucagonaemia, and (2) part of the unidentified diabetogenic signal in the foregut hypothesis may be glucagon itself or one or more glucagonotropic signals released from the proximal part of the small intestine.

\section{Does diabetic hyperglucagonaemia originate from the proximal gut?}

It is well known that in many animals, such as the rat, dog and cat, the pancreas is not the only source of glucagon; in these animals glucagon has also been identified in the gastrointestinal tract [24]. Furthermore, glucagon has been shown to circulate after total pancreatectomy in man, and in these patients basal levels increase several fold following meals, suggesting gut-derivation [25]. In addition, studies on human gastrointestinal mucosa have revealed small amounts of glucagon [26]. In these studies a glucagon RIA directed against the C-terminus of the glucagon molecule (using an antibody with no cross-reaction with glicentin or oxyntomodulin) was employed. Thus, the thought that endocrine cells in the mucosa of the small intestine might secrete glucagon is not novel. However, whether diabetic hyperglucagonaemia stems from the gastrointestinal tract remains unknown.

Glucagon is a product of the proglucagon gene. Proglucagon is produced in pancreatic alpha cells and in endocrine $L$ cells in the intestinal mucosa. In pancreatic alpha cells, post-translational processing of proglucagon by prohormone convertase 2 (PC2) results in glucagon, glicentin-related pancreatic peptide (GRPP) and major proglucagon fragment, of which glucagon seems to be the only biologically active peptide [27]. In the L cells, proglucagon is processed by prohormone convertase 1 (PC1). This processing results in the incretin hormone GLP-1, glucagon-like peptide-2 (GLP-2), which plays a central role in the regulation of small intestinal growth, and glicentin, which is most likely biologically inactive [28]. The glucagon sequence, together with GRPP, resides embedded in the glicentin peptide.

In healthy people the anatomically differentiated processing is sharply divided and follows the specific production of PC1 (L cells) and PC2 (alpha cells), respectively. L cells are predominantly located in the distal part of the small intestine [29], but recent studies have shown that endocrine cells capable of producing both incretin hormones, glucosedependent insulinotropic polypeptide (GIP) and GLP-1, exist in the mammalian small intestine, including the human duodenum [30]. These pluripotent cells are only sparsely described in the literature.

Interestingly, in total PC1 deficiency in humans exaggerated postprandial glucagon responses have been observed [31] and in PC1-deficient mice the processing of proglucagon to GLP-1 and GLP-2 is significantly reduced, whereas plasma glucagon levels are increased [32]. These observations suggest that $\mathrm{L}$ cells are able to process proglucagon to glucagon, possibly by using PC2. This notion is supported by the finding that an L cell model, the GLUTag cell line, secretes glucagon in addition to GLP-1 [33]; however, the mechanisms underlying this dual secretion pattern have not been established.

A shift from PC1 processing (GLP-1 production) to PC2 processing (glucagon production) in 'diabetic' proximal L cell-like cells would probably make these cells secrete glucagon at the cost of GLP-1 secretion, and, therefore, not only explains the postprandial hyperglucagonaemia in type 2 diabetes, but also another trait of the disease: postprandial hyposecretion of GLP-1 [34]. Thus, a changed processing of proglucagon in proximal small intestinal $\mathrm{L}$ cells might be the underlying basis of postprandial hyperglucagonaemia and postprandial hyposecretion of GLP-1, both considered to be significant contributors to the postprandial hyperglycaemia in type 2 diabetes. This hypothesis would allot glucagon itself a role in the puzzle of the unidentified diabetogenic factor in the foregut hypothesis.

Accordingly, it would be expected that bypass of the duodenum and proximal jejunum results in decreased glucagon secretion, which according to results of Shah et al. [18] would be expected to have a significant impact on diabetic glucose tolerance. However, glucagon secretion is affected by a number of gastrointestinal factors (see below) and the exact mechanisms behind changes in plasma glucagon concentrations are difficult to establish. Interestingly, a recently published longitudinal investigation has shown that fasting plasma glucagon is reduced progressively (up to 12 months) following RYGB [35]. Whether this change in baseline levels affects postprandial responses remains to be established. Nevertheless, in a cross-sectional study performed in three groups of obese women who had undergone either RYGB or LAGB or who acted as overweight controls, glucagon levels were found to be significantly reduced following a liquid meal (time point $180 \mathrm{~min}$ ) in the RYGB group [36]. Furthermore, reduced glucagon responses to OGTTs have been observed in GK diabetic rats following DJB [37]. In line with these observations, reduced hepatic glucose production (or endogenous glucose production) has been reported in individuals treated with RYGB [38]. However, the available data on glucagon secretion following RYGB are sparse, and it should 
be emphasised that elevated glucagon levels have also been observed following RYGB [39].

In addition to secretion of glucagon from intestinal endocrine cells, indirect intestine-derived mechanisms (elicited by glucose in the gastrointestinal tract and not by i.v. glucose) can be thought to be responsible for the hyperglucagonaemia in response to oral glucose.

\section{Indirect intestine-derived mechanisms}

As opposed to the glucagonostatic properties of GLP-1, the other incretin hormone, GIP, has been shown to stimulate pancreatic glucagon secretion [40]. Likewise, GLP-1's sister hormone, the other L cell hormone GLP-2, which is secreted in parallel (and in equimolar amounts) with GLP-1, has been shown to stimulate glucagon secretion from pancreatic alpha cells [41]. In healthy individuals one must assume that the glucagon-inhibiting effects of GLP-1 and insulin outweigh the glucagonotropic effects of GIP and GLP-2 following ingestion of nutrients, a disequilibrium resulting in glucagon suppression following ingestion of, for example, glucose. Changes in this (dis)equilibrium would inflict the level of glucagon suppression following oral glucose administration. Hence, lack of postprandial glucagon suppression in type 2 diabetes could theoretically be caused by increased postprandial secretion or glucagonotropic effects of GIP and/or GLP-2 on one scale pan and/ or by a reduced postprandial secretion or glucagonostatic effect of GLP-1 on the other side of the scale.

As mentioned, in some studies, postprandial levels of GLP-1 have been shown to be reduced in patients with type 2 diabetes compared with matched healthy control individuals [34]. Bearing GLP-1's glucagonostatic properties in mind this phenomenon would in itself be thought to contribute to a reduced suppression of postprandial glucagon secretion. However, hyposecretion of GLP-1 implies hyposecretion of its glucagonotropic sister peptide GLP-2; a hyposecretion which would be believed, to some extent, to counteract blunted GLP-1-induced glucagon suppression. Furthermore, in our studies showing the paradoxical regulation of glucagon responses to oral and isoglycaemic i.v. glucose no differences in GLP-1 responses to oral glucose between patients with type 2 diabetes and healthy volunteers were observed $[20,21]$. Thus, reduced/exaggerated responses of GLP-1/GLP-2 (if any) in patients with type 2 diabetes do not seem to explain the lack of glucagon suppression following oral glucose in these patients.

Under normal circumstances GIP, which is secreted from endocrine $\mathrm{K}$ cells (predominantly located in the mucosa of the duodenum and proximal jejunum) in response to nutrient ingestion, exerts a strong glucose-dependent insulinotropic effect on pancreatic beta cells and has, like
GLP-1, been shown to stimulate beta cell growth [42]. However, GIP administration has, unlike GLP-1 administration, been shown to enhance glucagon release in humans [43]. Thus, GIP appears to act like a double-edged sword with regard to blood sugar levels; on the one hand being able to reduce them (insulinotropic effect) and on the other hand being able to increase them (glucagonotropic effect); the latter most likely occurs during low blood sugar concentrations. Interestingly, human GIP has lost its insulinotropic power in type 2 diabetes [44], leaving it as a primarily glucagonotropic hormone in these patients. What would be the anticipated effect of bariatric surgery on GIP secretion? Obviously, surgically introduced bypass of nutrients from the proximal part of the small intestine (e.g. by RYGB), where GIP-secreting $\mathrm{K}$ cells reside in the highest numbers, would be thought to result in reduced GIP responses following ingestion of nutrients (Fig. 1). This is supported by the finding that blunted postprandial GIP secretion is associated with RYGB as opposed to normal GIP secretion following LAGB [36], and that obese patients with type 2 diabetes undergoing RYGB experience significant postoperative reductions in GIP levels alongside their resolution of diabetes [45]. Likewise, postprandial GIP levels decrease in obese patients following surgery that involves bypass of the $\mathrm{K}$ cell-rich proximal part of the small intestine [36, 46-48]. However, in other studies GIP responses to oral glucose have been shown to be increased 1 month following RYGB [10, 39], but this effect did not persist a year postoperatively [49].

Thus, reduced glucagonotropic GIP signalling may contribute to the restoration of normal glucose tolerance following surgically introduced bypass of the foregut in diabetic patients. Interestingly, GIP has also been implicated in the RYGBinduced resolution of type 2 diabetes via its effects linking consumption of high fat diets and the development of obesity, insulin resistance and type 2 diabetes [50]. As such, GIP could be a possible candidate for the unidentified 'diabetogenic factor' in the foregut hypothesis, as an intestinederived glucagonotropic and obesity-promoting signal.

\section{Summary and conclusion}

In addition to the restrictive elements incurred by bariatric surgical procedures (resulting in reduced food intake and weight loss) there is little doubt that the re-routing of nutrients (bypass of the duodenum and the proximal jejunum) brings about significant endocrine changes in the gastrointestinal system that contribute to the glucose-lowering effects of these operations. The hindgut hypothesis states that surgical rerouting of nutrients to the distal part of the small intestine, where GLP-1-secreting L cells are abundant, results in increased GLP-1 secretion and the concomitant glucose- 
lowering effects of GLP-1; whereas the foregut hypothesis emphasises that surgical bypass of the duodenum and proximal jejunum prevents the release of a putative diabetogenic signal, thereby causing resolution of type 2 diabetes. There is little doubt that increased GLP-1 signalling brings about glucose-lowering effects and since GLP-1 secretion has been shown consistently to be increased following these operations the 'hindgut hypothesis' has more or less become the 'hindgut dogma'. Contrary to this, the putative diabetogenic signal of the foregut hypothesis remains unidentified. It is well known that hyperglucagonaemia contributes to the hyperglycaemia characterising patients with type 2 diabetes, and recent studies from the group of the author suggest glucagon itself may be a candidate for the hitherto unknown diabetogenic signal of the foregut hypothesis. In the present paper it is hypothesised that diabetic hypersecretion of glucagon originates directly and/or indirectly (via glucagonotropic signals, e.g. GIP) from the mucosa of the proximal small intestine upon stimulation with nutrients. Thus, circumvention of the 'foregut' (duodenum and proximal jejunum) incurred by RYGB can be thought to lead to non-release of a diabetogenic signal, part of which may be the glucagonotropic and obesity-promoting intestinal hormone GIP, another part of which may be gut-derived glucagon and/or an unknown gut hormone. This, in combination with increased nutrient delivery to the hindgut and concomitant increased secretion of the insulinotropic and glucagonostatic intestinal hormone GLP-1 (the hindgut hypothesis), might constitute some of the endocrine changes responsible for the impressive proportion of patients being cured of type 2 diabetes following RYGB.

Ongoing studies investigating (1) whether endocrine cells in the mucosa of the RYGB-evaded part of the foregut are able to produce and secrete glucagon, and (2) the exact impact of GIP (and GLP-1 and GLP-2) on glucagon secretion will hopefully bring about a more detailed explanation, but so far there is little doubt that type 2 diabetes has to be regarded not only as a pancreatic disease, but also as a gastrointestinal disease entity.

Duality of interest The author declares that there is no duality of interest associated with this manuscript.

\section{References}

1. Pories WJ, Swanson MS, MacDonald KG et al (1995) Who would have thought it? An operation proves to be the most effective therapy for adult-onset diabetes mellitus. Ann Surg 222:339-350

2. Mason EE (2005) The mechanisms of surgical treatment of type 2 diabetes. Obes Surg 15:459-461

3. Rubino F, Gagner M (2002) Potential of surgery for curing type 2 diabetes mellitus. Ann Surg 236:554-559

4. Fehmann HC, Goke R, Goke B (1995) Cell and molecular biology of the incretin hormones glucagon-like peptide-1 and glucosedependent insulin releasing polypeptide. Endocr Rev 16:390-410
5. Wang Y, Perfetti R, Greig NH et al (1997) Glucagon-like peptide1 can reverse the age-related decline in glucose tolerance in rats. J Clin Invest 99:2883-2889

6. Buteau J, El-Assaad W, Rhodes CJ, Rosenberg L, Joly E, Prentki M (2004) Glucagon-like peptide-1 prevents beta cell glucolipotoxicity. Diabetologia 47:806-815

7. Larsson H, Holst JJ, Ahren B (1997) Glucagon-like peptide-1 reduces hepatic glucose production indirectly through insulin and glucagon in humans. Acta Physiol Scand 160:413-422

8. Holst JJ (2007) The physiology of glucagon-like peptide 1 . Physiol Rev 87:1409-1439

9. Borg CM, Le Roux CW, Ghatei MA, Bloom SR, Patel AG, Aylwin SJ (2006) Progressive rise in gut hormone levels after Roux-en-Y gastric bypass suggests gut adaptation and explains altered satiety. Br J Surg 93:210-215

10. Laferrere B, Heshka S, Wang K et al (2007) Incretin levels and effect are markedly enhanced 1 month after Roux-en-Y gastric bypass surgery in obese patients with type 2 diabetes. Diabetes Care 30:1709-1716

11. Le Roux CW, Aylwin SJ, Batterham RL et al (2006) Gut hormone profiles following bariatric surgery favor an anorectic state, facilitate weight loss, and improve metabolic parameters. Ann Surg 243:108-114

12. Naslund E, Gryback P, Hellstrom PM et al (1997) Gastrointestinal hormones and gastric emptying 20 years after jejunoileal bypass for massive obesity. Int J Obes Relat Metab Disord 21:387-392

13. Hickey MS, Pories WJ, MacDonald KG Jr et al (1998) A new paradigm for type 2 diabetes mellitus: could it be a disease of the foregut? Ann Surg 227:637-643

14. Pories WJ, Albrecht RJ (2001) Etiology of type II diabetes mellitus: role of the foregut. World J Surg 25:527-531

15. Rubino F, Forgione A, Cummings DE et al (2006) The mechanism of diabetes control after gastrointestinal bypass surgery reveals a role of the proximal small intestine in the pathophysiology of type 2 diabetes. Ann Surg 244:741-749

16. Rubino F, Marescaux J (2004) Effect of duodenal-jejunal exclusion in a non-obese animal model of type 2 diabetes: a new perspective for an old disease. Ann Surg 239:1-11

17. Unger RH, Orci L (1981) Glucagon and the A cell: physiology and pathophysiology (second of two parts). N Engl J Med 304:1575-1580

18. Shah P, Vella A, Basu A, Basu R, Schwenk WF, Rizza RA (2000) Lack of suppression of glucagon contributes to postprandial hyperglycemia in subjects with type 2 diabetes mellitus. J Clin Endocrinol Metab 85:4053-4059

19. Kirk RD, Dunn P, Smith JR, Beaven DW, Donald RA (1975) Abnormal pancreatic alpha-cell function in first-degree relatives of known diabetics. J Clin Endocrinol Metab 40:913-916

20. Knop FK, Vilsboll T, Madsbad S, Holst JJ, Krarup T (2007) Inappropriate suppression of glucagon during OGTT but not during isoglycaemic i.v. glucose infusion contributes to the reduced incretin effect in type 2 diabetes mellitus. Diabetologia 50:797-805

21. Knop FK, Vilsboll T, Hojberg PV et al (2007) Reduced incretin effect in type 2 diabetes: cause or consequence of the diabetic state? Diabetes 56:1951-1959

22. Muscelli E, Mari A, Casolaro A et al (2008) Separate impact of obesity and glucose tolerance on the incretin effect in normal subjects and type 2 diabetic patients. Diabetes 57:1340-1348

23. Meier JJ, Deacon CF, Schmidt WE, Holst JJ, Nauck MA (2007) Suppression of glucagon secretion is lower after oral glucose administration than during intravenous glucose administration in human subjects. Diabetologia 50:806-813

24. Holst JJ (1978) Extra-pancreatic glucagons. Digestion 17:168-190

25. Holst JJ, Pedersen JH, Baldissera F, Stadil F (1983) Circulating glucagon after total pancreatectomy in man. Diabetologia 25:396-399 
26. Baldissera FGA, Holst JJ (1984) Glucagon-related peptides in the human gastrointestinal mucosa. Diabetologia 26:223-228

27. Dhanvantari S, Seidah NG, Brubaker PL (1996) Role of prohormone convertases in the tissue-specific processing of proglucagon. Mol Endocrinol 10:342-355

28. Bell GI, Sanchez PR, Laybourn PJ, Najarian RC (1983) Exon duplication and divergence in the human preproglucagon gene. Nature 304:368-371

29. Eissele R, Goke R, Willemer S et al (1992) Glucagon-like peptide- 1 cells in the gastrointestinal-tract and pancreas of rat, pig and man. Eur J Clin Invest 22:283-291

30. Theodorakis MJ, Carlson O, Michopoulos S et al (2006) Human duodenal enteroendocrine cells: source of both incretin peptides, GLP-1 and GIP. Am J Physiol Endocrinol Metab 290:E550-E559

31. Jackson RS, Creemers JWM, Farooqi IS et al (2003) Smallintestinal dysfunction accompanies the complex endocrinopathy of human proprotein convertase 1 deficiency. J Clin Invest 112:1550-1560

32. Ugleholdt R, Zhu XR, Deacon CF, Orskov C, Steiner DF, Holst JJ (2004) Impaired intestinal proglucagon processing in mice lacking prohormone convertase 1. Endocrinology 145:1349-1355

33. Drucker DJ, Jin T, Asa SL, Young TA, Brubaker PL (1994) Activation of proglucagon gene transcription by protein kinase-A in a novel mouse enteroendocrine cell line. Mol Endocrinol 8: $1646-1655$

34. Toft-Nielsen MB, Damholt MB, Madsbad S et al (2001) Determinants of the impaired secretion of glucagon-like peptide- 1 in type 2 diabetic patients. J Clin Endocrinol Metab 86:3717-3723

35. Swarbrick MM, Stanhope KL, Austrheim-Smith IT et al (2008) Longitudinal changes in pancreatic and adipocyte hormones following Roux-en-Y gastric bypass surgery. Diabetologia 51: 1901-1911

36. Korner J, Bessler M, Inabnet W, Taveras C, Holst JJ (2007) Exaggerated glucagon-like peptide-1 and blunted glucosedependent insulinotropic peptide secretion are associated with Roux-en-Y gastric bypass but not adjustable gastric banding. Surg Obes Relat Dis 3:597-601

37. Pacheco D, de Luis DA, Romero A et al (2007) The effects of duodenal-jejunal exclusion on hormonal regulation of glucose metabolism in Goto-Kakizaki rats. Am J Surg 194:221-224

38. Klein S, Mittendorfer B, Eagon JC et al (2006) Gastric bypass surgery improves metabolic and hepatic abnormalities associated with nonalcoholic fatty liver disease. Gastroenterology 130: 1564-1572
39. Laferrere B, Teixeira J, McGinty J et al (2008) Effect of weight loss by gastric bypass surgery vs hypocaloric diet on glucose and incretin levels in patients with type 2 diabetes. J Clin Endocrinol Metab 93:2479-2485

40. Meier JJ, Gallwitz B, Siepmann N et al (2003) Gastric inhibitory polypeptide (GIP) dose-dependently stimulates glucagon secretion in healthy human subjects at euglycaemia. Diabetologia 46: 798-801

41. Meier JJ, Nauck MA, Pott A et al (2006) Glucagon-like peptide 2 stimulates glucagon secretion, enhances lipid absorption, and inhibits gastric acid secretion in humans. Gastroenterology 130:44-54

42. Baggio LL, Drucker DJ (2007) Biology of incretins: GLP-1 and GIP. Gastroenterology 132:2131-2157

43. Meier JJ, Gallwitz B, Siepmann N et al (2003) Gastric inhibitory polypeptide (GIP) dose-dependently stimulates glucagon secretion in healthy human subjects at euglycaemia. Diabetologia 46: 798-801

44. Nauck MA, Heimesaat MM, Orskov C, Holst JJ, Ebert R, Creutzfeldt W (1993) Preserved incretin activity of glucagonlike peptide 1 [7-36 amide] but not of synthetic human gastric inhibitory polypeptide in patients with type- 2 diabetes mellitus. J Clin Invest 91:301-307

45. Rubino F, Gagner M, Gentileschi P et al (2004) The early effect of the Roux-en-Y gastric bypass on hormones involved in body weight regulation and glucose metabolism. Ann Surg 240:236-242

46. Guidone C, Manco M, Valera-Mora E et al (2006) Mechanisms of recovery from type 2 diabetes after malabsorptive bariatric surgery. Diabetes 55:2025-2031

47. Jorde R, Burhol PG, Johnson JA (1981) The effect of jejunoileal bypass on postprandial release of plasma gastric inhibitory polypeptide (GIP). Scand J Gastroenterol 16:313-319

48. Sirinek KR, O'Dorisio TM, Hill D, McFee AS (1986) Hyperinsulinism, glucose-dependent insulinotropic polypeptide, and the enteroinsular axis in morbidly obese patients before and after gastric bypass. Surgery 100:781-787

49. Bose M, Olivan B, Teixeira J, Pi-Sunyer FX, Laferrere B (2009) Do incretins play a role in the remission of type 2 diabetes after gastric bypass surgery: what are the evidence? Obes Surg 19:217-229

50. Irwin N, Flatt PR (2009) Evidence for beneficial effects of compromised gastric inhibitory polypeptide action in obesityrelated diabetes and possible therapeutic implications. Diabetologia. doi:10.1007/s00125-009-1422-8 